\title{
Múltiples lesiones en parénquima cerebral debido a neurocisticercosis en una nativa de altura: reporte de caso
}

\section{Multiple lesions in the brain parenchyma due to neurocysticercosis in a high altitude native person: Report of a case}

\section{Correspondencia}

Douglas López de Guimaraes

douglasdeguimaraes25@yahoo.com

\section{Recibido: 23/02/2017}

Arbitrado por pares

Aprobado: 15/03/2017

Citar como: López-de Guimaraes D, Mezarina-Valverde J, Flores-Plata J, Pajuelo-Vera M.. Múltiples lesiones en parénquima cerebral debido a neurocisticercosis en una nativa de altura: reporte de caso. Acta Med Peru. 2017;34(1):57-60

\author{
Douglas López-de Guimaraes ${ }^{1,2}$, Jorge Eduardo Mezarina-Valverde ${ }^{3}$, Jorge \\ Antonio Flores-Plata ${ }^{4}$, Margarita Amelia Pajuelo-Vera ${ }^{5,2}$ \\ 1 Departamento de Medicina del Hospital Víctor Ramos Guardia. Huaraz, Perú. \\ 2 Universidad Nacional de Ancash Santiago Antúnez de Mayolo. Huaraz, Perú. \\ 3 Departamento de Emergencia del Hospital Víctor Ramos Guardia. Huaraz, Perú. \\ 4 Unidad de Cuidados Intensivos del Hospital Víctor Ramos Guardia. Huaraz, Perú. \\ 5 Departamento de Pediatría del Hospital Víctor Ramos Guardia. Huaraz, Perú.
}

\section{RESUMEN}

Se reporta un caso de neurocisticercosis parenquimatosa activa múltiple observado en una paciente mujer de 19 años, natural de Acobamba (2 $518 \mathrm{~m}$ de altitud), Yungay-Ancash, residente en área rural, criadora de cerdos; refirió la presencia cefalea intermitente desde hace cuatro años, negó haber padecido convulsiones. Un día antes del ingreso presentó cefalea, náuseas, vómitos, trastorno de conciencia y agitación, por lo que fue referida al Hospital Víctor Ramos Guardia de Huaraz. Mediante el estudio por imágenes, la tomografía cerebral sin contraste mostró la presencia de múltiples imágenes hipodensas que en su interior presentaban imágenes hiperdensas que corresponderían a escólex de cisticerco; además, algunas lesiones se acompañaban de edema. Los demás exámenes auxiliares fueron negativos. Se procedió primero a reducir la hipertensión endocraneana y posteriormente se indicó uso de albendazol $800 \mathrm{mg}$ por día durante 14 días. En la evaluación de control al mes del alta la paciente estaba asintomática.

Palabras clave: Neurocisticercosis; Zoonosis; Ecosistema andino (fuente: DeCS/BIREME).

\section{ABSTRACT}

A case of active multiple neurocysticercosis affecting the brain parenchyma is reported. This occurred in a 19 year-old woman born in Acobamba (2,518 meters above sea level), Yungay Ancash, living in a rural area, who used to work in a farm breeding swine. The complained of intermittent headaches for the last four years. She never had seizures. One day before admission she presented with headache, nausea, vomiting, consciousness alteration, and agitation, so she was referred to Victor Ramos-Guardia Hospital in Huaraz. A CT scan of the head with no contrast substance revealed the presence of multiple hypodense images with some hyperdense structures inside of them corresponding to cysticercal scolices. Also, some lesions had edema surrounding them. Other testes were reported as negative. The first step in management was to reduce cranial hypertension and later albendazole, $800 \mathrm{mg} /$ day was instituted, for 14 days. In the control assessment performed one month later, the patient was asymptomatic.

Keywords: Neurocysticercosis; Zoonoses; Andean ecosystem (source: MeSH/NLM). 


\section{INTRODUCCIÓN}

La Taenia solium es un céstodo zoonótico que produce dos enfermedades en el ser humano: teniasis en su fase adulta y cisticercosis en la fase quística larvaria. La Organización Mundial de la Salud considera que la neurocisticercosis es una enfermedad tropical desatendida, y estima que a nivel mundial afecta alrededor de 50 millones de personas, muchos pueden tornarse sintomáticos en algún momento de sus vidas y 50000 fallecen anualmente; también es responsable de más del 50\% de los casos de epilepsia de inicio tardío en los países en vías de desarrollo ${ }^{[1,2]}$. Una reciente revisión sistemática reportó que en Latinoamérica la infección activa por Taenia solium fue de 4,08\% $(0,94$ a 9,12\%), la seroprevalencia por anticuerpos de 13,03\% $(1,82$ a $31,22 \%)$ y la prevalencia de teniasis varió entre o al $17,25 \%$ [3]. Las tasas más elevadas de cisticercosis porcina corresponden a Guatemala, Honduras, México y Perú ${ }^{[1]}$.

La neurocisticercosis es una infección parasitaria del sistema nervioso central y sus cubiertas meníngeas por el estadio larvario de Taenia solium. Este proceso neurológico es endémico en la mayoría de países en vías de desarrollo de Latinoamérica, Haití, África subsahariana y grandes regiones del continente asiático que incluyen a la India, sudeste del Asia, Indonesia y China ${ }^{[4,5]}$. Es una enfermedad emergente en los países desarrollados debido al incremento de la inmigración de personas provenientes de las zonas endémicas ${ }^{[6]}$. En el cerebro se presenta en dos formas: parenquimatosa (sustancia gris cerebral) y extraparenquimatosa o racemosa (intraventricular, subaracnoideo, intraoculary espinal) [7]. Presentamos un caso de neurocisticercosis parenquimatosa activa con quistes múltiples observado en nativo de altura, en el Hospital Víctor Ramos Guardia de Huaraz - Ancash.

\section{REPORTE DE CASO}

Mujer de 19 años de edad, natural y procedente del Centro Poblado Acobamba ( 2518 de altitud), ubicado en la zona rural de Yungay-Ancash, ama de casa, madre soltera de un hijo de 18 meses, católica, con estudios primarios concluidos, que se dedicaba a la crianza de cerdos. Ingresó al servicio de emergencia del Hospital Víctor Ramos Guardia de Huaraz referida del hospital de Yungay por presentar trastorno de conciencia, cefalea y vómitos. Según hermano, la paciente sufría de cefalea intermitente desde hace 4 años. Un día antes del ingreso, la paciente presentó cefalea más intensa acompañada de náuseas y vómitos; luego se tornó somnolienta, sin respuesta al llamado, con agitación ocasional, sin poder sostenerse en pie ni realizar sus actividades diarias. No hubo ni convulsiones ni alza térmica. Funciones biológicas conservadas.

Al ingreso a emergencia, la paciente no respondía al interrogatorio y mostraba agitación ocasional. Ventilaba espontáneamente. No hubo presencia de ictericia, edemas ni adenomegalias. Al examen físico se encontró hipotensión (tensión arterial: 88/40mmHg), bradicardia (frecuencia cardiaca: 56 latidos por minuto), saturación de $\mathrm{O}_{2}$ al $90 \%$. Frecuencia respiratoria y temperatura dentro de rangos normales. Peso: $50 \mathrm{~kg}$. Reflejos osteotendinosos conservados. Tórax: mamas con escasa secresión láctea.
Pulmones: regular paso del murmullo vesicular, no crépitos. Sistema cardiovascular: ruidos cardíacos rítmicos, tendencia a la bradicardia, no soplos. Abdomen: blando, depresible, no visceromegalia. Sistema Neurológico: pupilas isocóricas y fotoreactivas, no déficit motor, impresiona leve rigidez de nuca, Glasgow: $12(3 / 4,4 / 5,5 / 6)$. Resto de los sistemas, no contributorio. Se planteó como diagnóstico trastorno del sensorio: hemorragia subaracnoidea vs. meningitis aguda.

En los exámenes de laboratorio de ingreso se tuvo: hemoglobina: $11.8 \mathrm{~g} / \mathrm{dL}$, hematocrito: $38 \%$, leucocitos: $14200 / \mathrm{mm}^{3}$. (abastonados: 18\%, segmentados: 70\%, eosinófilos: 0\%, basófilos: $0 \%$, monocitos: $2 \%$, linfocitos: 10\%), glucosa: $107 \mathrm{mg} / \mathrm{dL}$, urea: $20 \mathrm{mg} / \mathrm{dL}$; creatinina: $0.5 \mathrm{mg} / \mathrm{dL}$. Lactato deshidrogenasa: $364 \mathrm{U} / \mathrm{L}$, volumen de sedimentación glomerular: $18 \mathrm{~mm} / \mathrm{h}$, proteína $C$ reactiva: $48 \mathrm{mg} / \mathrm{dL}$, aglutinaciones: negativo. Frotis de sangre periférica para Bartonela: negativo. Exámenes de orina, gases arteriales, electrolitos y radiografía del tórax dentro de límites normales. No se realizó examen de heces. En la tomografía (TAC) sin contraste se identificaron múltiples imágenes hipodensas que en su interior presentaban imágenes hiperdensas que corresponderían a escólex de cisticerco, resultados concordantes con el diagnóstico de neurocisticercosis parenquimatosa activa múltiple (Figura 1). La ecografía abdominal completa, tomada en día posterior al ingreso, no mostró alteraciones morfológicas.

Se inició el manejo terapéutico en la unidad de shock trauma. Se administró oxígeno por catéter nasal, $\mathrm{NaCl}$ al 0,9\% por vía endovenosa, además de prescripción de ceftriaxona, manitol, dexametasona, fenitoína, haloperidol, soporte médico y de enfermería. Dos días después del ingreso pasó a hospitalización en sala de medicina y al día siguiente se inició terapia con albendazol $800 \mathrm{mg} /$ día por 14 días. Cinco días después del ingreso, a solicitud de la paciente, se la trasladó a Hospital de Yungay. Tres días después, fue evaluada por medicina en hospital de Huaraz, la paciente estuvo asintomática.

\section{DISCUSIÓN}

El caso presentado es consistente con neurocisticercosis parenquimatosa activa múltiple, cuadro que no es raro en nuestro medio andino, especialmente cuando proceden de comunidades rurales. Llamó la atención que nuestro paciente presentara múltiples lesiones intracraneales, a diferencia de lo mostrado por otros autores ${ }^{[8]}$. Aunque la paciente no presentó convulsiones, sí se observaron signos de hipertensión endocraneana (cefalea, náuseas, vómitos y trastorno de conciencia), razón por la que se decidió iniciar el manejo con manitol y dexametasona durante tres días con el fin de reducir el edema cerebral, antes de comenzar el tratamiento antihelmíntico con albendazol.

El ser humano es el único huésped definitivo de la tenia adulta, mientras que la forma larvaria (cisticerco) puede residir tanto en humanos como cerdos. En el ciclo de transmisión, algunos proglótidos grávidos son eliminados con las heces por el humano. Cada proglótido libera miles de huevos infectantes 

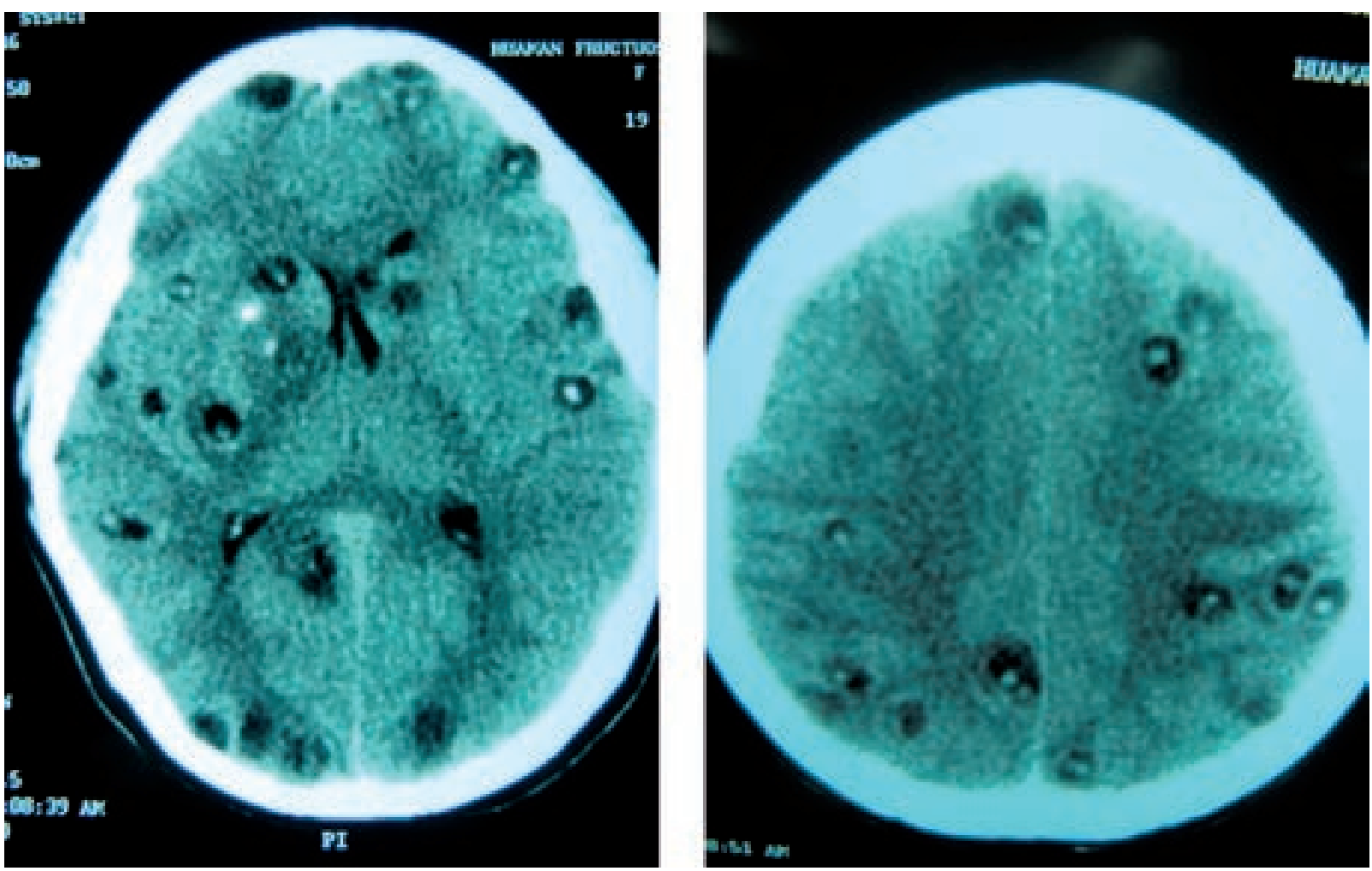

Figura 1. La TAC del cerebro sin contraste muestra múltiples imágenes hipodensas con imágenes hiperdensas en su interior, compatibles con larvas de Taenia solium o cisticercos. Este es el único hallazgo patognomónico de neurocisticercosis (10).

al medio ambiente; en áreas donde la disposición de heces es deficiente, los cerdos ingieren los huevos de Taenia solium. En el tracto intestinal del cerdo los huevos liberan oncosferas que atraviesan la barrera intestinal llegando al torrente sanguíneo para localizarse en cualquier parte de los tejidos del animal donde maduran en metacestodos y luego en larvas (cisticercos) en un tiempo promedio de tres a cuatro meses (cisticercosis porcina). El ciclo biológico se completa cuando el humano consume la carne porcina, parasitada con cisticercos, insuficientemente cocida, y que luego de ser liberada por acción de las enzimas digestivas se ancla en la pared del intestino delgado y los proglótidos comienzan a multiplicarse hasta alcanzar su forma adulta en unos cuatro meses ${ }^{[2,9,10]}$.

La presentación clínica inicial de neurocisticercosis se produce por tres mecanismos principales: por la presencia física del parásito (efecto de masa, obstrucción), la consecuente respuesta inflamatoria con edema perilesional y la presencia de tejido cicatrizal residual (fibrosis, granulomas y calcificaciones) ${ }^{[1]}$. En muchos casos la infección es asintomática y suele identificarse como un hallazgo imagenológico (por TAC o resonancia magnética [RM]). Las manifestaciones clínicas dependen de diversos factores como el número, estadio y localización de los cisticercos en el sistema nervioso, y de la severidad de la respuesta del huésped a la infección. La neurocisticercosis puede producir casi cualquier síntoma neurológico; la epilepsia de inicio tardío (sobre todo en mayores de 25 años) y la hipertensión intracraneal son las manifestaciones clínicas más comunes ${ }^{[10,12]}$. La convulsión ocurre en el 70 a $80 \%$ de los casos sintomáticos y puede aparecer en cualquier etapa del ciclo biológico del cisticerco, desde quistes viables a calcificaciones. Los quistes viables inducen convulsiones por un efecto de compresión sobre el parénquima cerebral, los quistes coloidales y granulares lo hacen como resultado de la reacción inflamatoria que se desencadena por el ataque del parásito al sistema inmune del huésped, y en las lesiones calcificadas, por la gliosis que se desarrolla alrededor de los parásitos muertos, así como la exposición del material antigénico al parénquima cerebral o incluso el desarrollo de esclerosis del hipocampo ${ }^{[7,10]}$.

El cisticerco se desarrolla en cuatro estadios que tienen diferente apariencia en la neuroimagen: vesicular, que contiene la larva viva; coloidal, que contiene la larva en degeneración; nodular granular, donde se observa engrosamiento de la membrana del quiste y el de calcificación. Solamente los quistes en estadio vesiculary coloidal contienen la larva viva y ameritan tratamiento anticisticercósico ${ }^{[13]}$. El tratamiento de la neurocisticercosis depende de la agudeza y severidad de la presentación clínica, el grado de compromiso, el estadio de degeneración del quiste, la inflamación y las potenciales complicaciones. Comprende medidas inmediatas para prevenir la morbimortalidad (drogas antiepilépticas, corticoides, medicamentos antihelmínticos y procedimientos quirúrgicos) ${ }^{[14]}$. La combinación de albendazol y dexametasona o prednisolona debe considerarse para adultos y niños con neurocisticercosis del parénquima cerebral pues ambos disminuyen el número de lesiones activas en los estudios de neuroimagen (evidencia de nivel B) y reducen la frecuencia de convulsiones a largo plazo (evidencia de nivel $B$ ) ${ }^{[13]}$. Se debe 
preferir el uso de albendazol a praziquantel, tal como indica un metaanálisis desarrollado en el $2008^{[15]}$.

Los agentes antiparasitarios están indicados en el tratamiento de quistes viables; estos agentes cisticidas producen un incremento transitorio de la respuesta inflamatoria con deterioro clínico y convulsiones; por lo que también se utilizan los corticoides [16] para controlar la inflamación que se desencadena durante la desaparición de las lesiones ${ }^{[17]}$. La administración de dexametasona (dosis de $8 \mathrm{mg} / \mathrm{día}$ [3 mg, mañana y tarde; 2 mg, por la noche] por 21 días) mejoró el control de la inflamación y disminuyeron las convulsiones durante el tratamiento ${ }^{[18]}$. Recientemente, se reportó que la combinación de albendazol ( $15 \mathrm{mg} / \mathrm{kg} / \mathrm{dí}$ por 10 días) y praziquantel $(50 \mathrm{mg} / \mathrm{kg} / \mathrm{día}$ por 10 días) fue más eficaz que solo el albendazol en destruir los quistes múltiples de cisticercos viables en el cerebro humano ${ }^{[19]}$.

El análisis serológico de elección es el inmunoblot que tiene una sensibilidad del 94 al $98 \%$ y una especificidad del $100 \%$ en pacientes con dos o más quistes, o que tienen lesiones que realzan o se intensifican en la neuroimagen ${ }^{[7]}$. Los estudios de neuroimagen (TAC o RM) deben catalogarse como consistente, probable o diagnóstico. En el estadio vesicular hay una mínima reacción inflamatoria por lo que se observa una lesión quística intraparenquimal rodeada por una pared delgada e isodensa, el fluido también es isodenso, se aprecia también un nódulo claro en el interior del quiste que representa al escólex; no hay edema alrededor de la lesión ni un incremento de la captación cuando se usa sustancia de contraste. En los estudios con RM (tecnología que no existe actualmente en la sierra de Ancash), el quiste tiene señal idéntica al líquido cefalorraquídeo, mide de 10 a $20 \mathrm{~mm}$ y presenta un nódulo mural intraluminal que corresponde al escólex, que es el hallazgo patognomónico de este estadio. La RM es superior a la TAC para visualizar la anatomía, la estructura del cerebro y los quistes. La TAC es superior para detectar calcificaciones ${ }^{[20-22]}$.

Desde 1993 la cisticercosis se considera una enfermedad erradicable. Se han hecho revisiones de las bases conceptuales para el control y erradicación del complejo teniasis/cisticercosis, así como intervenciones a gran escala como el realizado en una ciudad de la costa norte, Tumbes ${ }^{[23]}$, y se ha demostrado que sí es posible interrumpir la transmisión entre humanos y cerdos, incluso en zonas de alta endemicidad ${ }^{[24]}$.

\section{REFERENCIAS BIBLIOGRÁFICAS}

1. Bouteille B. Épidémiologie de la cysticercose et de la neurocysticercose. Med Sante Trop. 2014;24(4):367-74.

2. Garcia HH, Nash TE, Del Bruto OH. Clinical symptoms, diagnosis, and treatment of neurocysticercosis. Lancet Neurol. 2014;13(12):1202-15.

3. Coral Almeida M, Gabriël S, Abatih EN, Praet N, Benitez W, Dorny P. Taenia solium human cisticercosis: A systematic review of seroepidemiological data from endemic zones around the world. Plos Negl Trop Dis. 2015;9(7):e0003919.

4. Winkler AS, Richter $\mathrm{H}$. Landscape analysis: management of neurocysticercosis with emphasis on - low and middle - incomes countries. Geneva: World Health Organization; 2015.
5. Fogang YF, Savadogo AA, Camara M, Toffa DH, Basse A, Sow AD, et al. Managing neurocysticercosis: challenges and solutions. Int J Gen Med. 2015;8:333-44.

6. Cantey PT, Coyle CM, Sorvillo FJ, Wilkins PP, Starr MC, Nash TE. Neglectec parasitic infections in the United States: Cysticercosis. Am J Trop Med Hyg. 2014;90(5):805-9.

7. Mahale RR, Metha A, Rangasetty S. Extraparenquimal (racemose) neurocysticercosis and its multitude manifestations: a comprehensive review. J Clin Neurol. 2015;11(3):203-11.

8. Del Brutto OH, Campos X. Massive neurocysticercosis: encephalitic versus non-encephalitic. Am J Trop Med Hyg. 2012;87(3):381.

9. Meza Lucas A, Aguilar Revoredo F. Teniasis humana por Taenia solium. Rev Mex Patol Clin. 2002;49(2):92-9.

10. Del Brutto OH. Neurocysticercosis. Neurohospitalist. 2014;4(4):205-12.

11. Kimura Hayama ET, Higuera JA, Corona Cedillo R, Chávez Macías $L_{\text {, }}$ Perochena A, Quiroz Rojas LY, et al. Neurocysticercosis: radiologicpathologic correlation. RadioGraphics. 2010;30(6):1705-19.

12. Saavedra $\mathrm{H}$, Gonzales I, Alvarado MA, Porras MA, Vargas $V$, Cjuno $\mathrm{RA}$, et al. Diagnóstico y manejo de la neurocisticercosis en el Perú. Rev Per Med Exp Salud Publica. 2010;27(4):586-91.

13. Baird RA, Wiebe S, Zunt JR, Halperin JJ, Gronseth G, Ross KL. Treatment of parenchymal neurocysticercosis. Neurology. 2013;80(15):1424-9.

14. NashTE, García HH. Diagnosis and treatment of neurocysticercosis. Nat Rev Neurol. 2011;7(10):584-9.

15. Matthaiou DK, Panos G, Adamidi ES, Falagas ME. Albendazole versus Praziquantel in the treatment of neurocysticercosis: a metaanalysis of comparative trials. Plos Negl Trop Dis. 2008;2(3):e194.

16. Nash TE, Mahanty S, Loeb JA, Theodore WH, Friedman A, Sander JW, et al. Neurocysticercosis: a natural human model of epileptogenesis. Epilepsia. 2015;56(2):177-83.

17. Cuello García CA, Roldán Benítez YM, Pérez Gaxiola G, Villareal Careaga J. Corticosteroids for neurocysticercosis: a systematic review and meta - analysis of randomized controlled trials. Int J Infect Dis. 2013;17(8):e583-92.

18. García HH, Gonzales I, Lescano AG, Bustos JA, Pretell JE, Saavedra $\mathrm{H}$, et al. Enhanced steroid dosing reduces seizures during antiparasitic treatment for cysticercosis and early after. Epilepsia. 2014;55(9):1452-9.

19. Garcia HH, Lescano AG, Gonzales I, Bustos JA, Pretell EJ, Horton J, et al. Cysticidal efficacy of combined treatment with Praziquantel and Albendazole for parenquimal brain cysticercosis. Clin Infect Dis. 2016;62(11):1375-9.

20. García HH, Del Brutto OH, Nash TE, White Jr AC, Tsang VCW, Gilman RH. New concepts in the diagnosis and management of neurocysticercosis (Taenia solium). Am J Trop Med Hyg. 2005;72(1):3-9.

21. Sarria Estrada S, Frascheri Verzelli L, Siurana Montilva S, Auger Acosta C, Rovira Cañellas A. Neurocisticercosis: hallazgos radiológicos. Radiología. 2013;55(2):130-41.

22. Venkat B, Aggarwal N, Makhaid S, Sood R. A comprehensive review of imaging findings in human cysticercosis. Jpn J Radiol. 2016;34(4):241-57.

23. Gilman GR, GonzalezAE, Llanos Zavalaga F, TsangVCW, García HH. Prevention and control of Taenia solium taeniasis/cysticercosis in Peru. Pathog Glob Health. 2012;106(5):312-8.

24. Garcia HH, Gonzales AE, Tsang VCW, O'Neal SE, Llanos Zavalaga $\mathrm{F}$, Gonzalvez $\mathrm{G}$, et al. Elimination of Taenia solium transmission in the Northern Peru. N Engl J Med. 2016;374(24):2335-44. 